\title{
First encounters of the microbial kind: perinatal factors direct infant gut microbiome establishment
}

\author{
Kevin Linehan ${ }^{1,2,3}$, Eugene M. Dempsey ${ }^{2,4}$, C. Anthony Ryan ${ }^{2,4}$, R. Paul Ross $^{2,3}$, Catherine Stanton ${ }^{1,2}$ \\ ${ }^{1}$ Teagasc Food Research Centre, Moorepark, Fermoy, Co. Cork P61 C996, Ireland. \\ ${ }^{2}$ APC Microbiome Ireland, Biosciences Institute, University College Cork, Lee Maltings, Cork, Cork T12 YT20, Ireland. \\ ${ }^{3}$ School of Microbiology, University College Cork, Cork T12 YN60, Ireland. \\ ${ }^{4}$ Department of Paediatrics \& Child Health and INFANT Centre, University College Cork, Cork T12 YN60, Ireland.
}

Correspondence to: Prof Catherine Stanton, Teagasc Food Research Centre, Moorepark, Moorepark West, Fermoy, Co. Cork P61 C996, Ireland. E-mail: catherine.stanton@teagasc.ie

How to cite this article: Linehan K, Dempsey EM, Ryan CA, Ross RP, Stanton C. First encounters of the microbial kind: perinatal factors direct infant gut microbiome establishment. Microbiome Res Rep 2022;1:10. https://dx.doi.org/10.20517/mrr.2021.09

Received: 2 Dec 2021 First Decision: 22 Dec 2021 Revised: 28 Dec 2021 Accepted: 11 Jan 2022 Published: 1 Mar 2022

Academic Editor: Marco Ventura Copy Editor: Xi-Jun Chen Production Editor: Xi-Jun Chen

\begin{abstract}
The human gut microbiome harbors a diverse range of microbes that play a fundamental role in the health and well-being of their host. The early-life microbiome has a major influence on human development and long-term health. Perinatal factors such as maternal nutrition, antibiotic use, gestational age and mode of delivery influence the initial colonization, development, and function of the neonatal gut microbiome. The perturbed early-life gut microbiome predisposes infants to diseases in early and later life. Understanding how perinatal factors guide and shape the composition of the early-life microbiome is essential to improving infant health. The following review provides a synopsis of perinatal factors with the most decisive influences on initial microbial colonization of the infant gut.
\end{abstract}

Keywords: Gut microbiome, neonatal microbiota, diseases, antibiotics, early programming, mode of birth, perinatal factors

\section{INTRODUCTION}

The microbiome depicts the microbiota and their "theatre of activity", encompassing the combined nucleic acids (including viruses and bacteriophages), structural components and microbial metabolites related to 
the microbiota ${ }^{[1]}$. The gut microbiota describes the trillions of microbial cells (bacteria, archaea, protists, and fungi) occupying the intestine, with Bacteroidetes and Firmicutes accounting for $90 \%$ of the total bacterial inhabitants and Actinobacteria, Cyanobacteria, Fusobacteria, Proteobacteria and Verrucomicrobia constituting the remaining $10 \%{ }^{[2]}$. These gut microbiota engage in a plethora of host biological responses, including the regulation of immunity ${ }^{[3]}$, energy homeostasis, protection against pathogens and bioactive metabolite production ${ }^{[4]}$. Furthermore, the disparities in the intestinal microbiota have been linked to disease states such as metabolic disorder ${ }^{[5]}$, cancer $^{[6]}$, and cardiovascular disease ${ }^{[7]}$. The initial formation of the gut microbiota is essential in maintaining intestinal homeostasis and symbiosis in neonates, shaping and guiding the development of the immune and nervous systems during a critical developmental window ${ }^{[8]}$. Although the mechanism at present is not fully elucidated, the current consensus is that a disrupted microbiota in early life can leave a lasting footprint on health in early life and beyond ${ }^{[9]}$. The first encounters of the neonatal gut with microbes are directed by intrinsic and extrinsic factors, collectively named "perinatal factors". These consist of maternal nutrition, antibiotic use, gestational age, genetics and mode of delivery ${ }^{[10]}$. Early-life gut microbiome imbalances predispose infants to colonisation by opportunistic pathogens, such as Enterococcus, Enterobacter, Clostridium, and Klebsiella species. Furthermore, infant gut dysbiosis can lead to impaired growth and an increased risk of sepsis and necrotizing enterocolitis (NEC), especially in preterm new-born $\mathbf{s}^{[1]}$. Longer-term consequences of perturbed microbial colonization include allergies, asthma, metabolic syndrome, diabetes and inflammatory bowel disease ${ }^{[12]}$. Therefore, it is essential to elucidate the extent to which perinatal factors may influence the microbial bond between mother and infant. Further understanding of how this intricate relationship may be influenced by perinatal factors is imperative to optimise mother and infant health in the perinatal period and inform the adequate type and timing of therapeutic interventions. In this short review, we discuss the core microbes which initially colonize the infant gut and decipher the influence of perinatal factors on directing these first encounters.

\section{CORE INFANT GUT MICROBIOME}

The first encounters of microbes with the infant intestine represent the de novo assembly of a complex microbial community ${ }^{[13]}$. In contrast to adults, the gut microbiome in early life is unstable, highly dynamic and low in diversity ${ }^{[14]}$. At birth, the infant gut is an aerobic environment, inhabited by Escherichia and Enterococcus. These facultative bacteria thrive on residual oxygen present, resulting in a lowered redox potential and thus shifting the infant gut to an anaerobic environment. Obligate anaerobes, including Firmicutes such as Clostridia, Bacteroidetes and the bedrock of the infant gut microbiome, bifidobacteria, begin to flourish at this point ${ }^{[15]}$. Bifidobacteria constitute up to $37 \%$ of the infant gut microbiota in early $\operatorname{life}^{[16]}$. Along with Veillonella, Streptococcus, Citrobacter, Escherichia, as well as Bacteroides and Clostridium, they dominate the core infant gut microbiome ${ }^{[17]}$. In Table 1, we summarize the characteristics and functions of these pioneering microbes in the infant gut. Despite large variance at the intra-individual level, a recent multi-population cohort meta-analysis revealed the existence of specific taxonomic patterns or infant community state types (ICSTs) ${ }^{[29]}$. This study subdivided ICSTs into four macro groups, $<1$ month of age, between 1 and 6 months of age, between 6 and 12 months of age and between 1 and 3 years of age. Higher microbial complexity in samples from infants aged between 12 and 36 months compared to those from children less than one-month-old was observed. This increase in biodiversity appeared to have caused a reduction in the relative abundance of the Bifidobacterium genus in favour of Bacteroides, Feacalibacterium, Blautia and Ruminococcus genera. ICSTs are further modulated by birth mode and feeding type, which will be discussed later in this review.

\section{PERINATAL FACTORS AND THE FIRST MICROBIAL ENCOUNTERS}

In this section, we discuss the effect of perinatal factors on the initial colonization of the infant gut with microbes (see Figure 1). The majority of studies on this topic relate the relative abundance of microbes in 
Table 1. Characteristics, functions and metabolites produced by the core infant gut microbiome members

\begin{tabular}{|c|c|c|c|}
\hline Microbes & Key functions \& characteristics in the infant gut & Microbial metabolites produced & Refs. \\
\hline Bifidobacteria & $\begin{array}{l}\text { Saccharolytic degradation of diet-derived glycans and host-provided carbohydrates, known as host glycans, } \\
\text { including mucins and human milk oligosaccharides (HMOs) }\end{array}$ & $\begin{array}{l}\gamma \text {-aminobutyric acid (GABA), conjugated linoleic acid (CLA), short } \\
\text { chain fatty acids (SCFA), Group B vitamins (B1, B3, B6, B9, B12) }\end{array}$ & {$[18-20]$} \\
\hline $\begin{array}{l}\text { Genus } \\
\text { Bacteroides }\end{array}$ & $\begin{array}{l}\text { Metabolize HMOs, mucins and complex plant polysaccharides (starch, cellulose, xylans, and pectins). } \\
\text { Proteolytic activity via extracellular proteases. Deconjugation of bile acids }\end{array}$ & $\begin{array}{l}\text { GABA, vitamin K2, SCFA, Group B vitamins (B1, B2, B5, B6, B7, B9, } \\
\text { B12) }\end{array}$ & [19-23] \\
\hline $\begin{array}{l}\text { Genus } \\
\text { Veillonella }\end{array}$ & $\begin{array}{l}\text { Produce propionate from end products of carbohydrate fermentation (e.g., lactate). Propionate displays } \\
\text { anti-inflammatory features, influences glucose and energy homeostasis and increases insulin sensitivity }\end{array}$ & SCFA & [24] \\
\hline $\begin{array}{l}\text { Genus } \\
\text { Streptococcus }\end{array}$ & $\begin{array}{l}\text { Specific members of the genus Streptococcus form part of the core infant gut microbiota and are among the } \\
\text { first established bacteria in the infant gut within the first } 24 \mathrm{~h} \text { following birth }\end{array}$ & Serotonin, GABA, histamine & [19] \\
\hline Genus Collinsella & $\begin{array}{l}\text { Present in large amounts when infant gut microbiota is dominated by bifidobacteria. Currently comprises } \\
\text { six species isolated from human faeces and vaginal tract }\end{array}$ & Group B vitamins (B6) & {$[20,25,26]$} \\
\hline $\begin{array}{l}\text { Genus } \\
\text { Lactobacillus }\end{array}$ & $\begin{array}{l}\text { Vertical transmission of Lactobacillus species presents the origin of infant Lactobacillus microbiota } \\
\text { component. It may be related to the } \mathrm{HMO} \text { metabolism of infants }\end{array}$ & $\begin{array}{l}\text { Serotonin, GABA, acetylcholine, histamine, CLA, SCFA, Group B } \\
\text { vitamins (B1, B2, B7, B9, B12) }\end{array}$ & {$[19,20,27]$} \\
\hline $\begin{array}{l}\text { Genus } \\
\text { Akkermansia }\end{array}$ & $\begin{array}{l}\text { A. muciniphila is the sole intestinal representative in the human gut from early life. May ferment HMOs. The } \\
\text { target for therapeutics aimed at increasing barrier function }\end{array}$ & Serotonin, SCFA & {$[28]$} \\
\hline
\end{tabular}

fecal samples to the composition of the gut microbiota, thus any changes or differences in microbial content discussed herein are based upon changes/differences in the relative abundance of microbes unless otherwise stated. While this review will focus on the establishment of bacteria in the infant gut, we acknowledge that these factors may also influence the infant gut virome ${ }^{[30]}$, mycobiome and sporobiota ${ }^{[31]}$.

\section{Maternal health status during pregnancy}

Maternal health during pregnancy is a key input in fetal health and infant development. Numerous studies have described aberrant gut colonization in infants born to mothers who suffered from elevated stress and cortisol levels throughout gestation ${ }^{[32]}$. Specifically, increased abundances of pathogenic bacteria Proteobacteria (Escherichia, Enterobacter, Serratia, Citrobacter, Campylobacter) and Firmicutes (Veillonella and Finegoldia) in addition to decreased abundances of beneficial bacteria such as lactic acid bacteria (Lactobacillus, Lactococcus, Aerococcus), Actinobacteria (bifidobacteria, Collinsella, Eggerthella) and Akkermansia have been noted ${ }^{[32]}$. While weight gain during pregnancy is a natural physiological response, excessive gestational weight gain (defined as $16 \mathrm{~kg}$ or above for women with BMI 19.8-25 or above $11.5 \mathrm{~kg}$ for women with BMI $>25^{[33]}$ has been reported to lead to the increased relative abundance of Escherichia and Dorea in vaginally born infants ${ }^{[34]}$. Metagenomic analysis has also shown that gestational weight gain impacts the function of the initial infant gut microbiome ${ }^{[35]}$. Excessive maternal weight gain during pregnancy has been associated with enrichment of phenylalanine, cysteine/serine, folate, thiamine, biotin, and pyridoxine synthesis pathways and bacterial glucose pathways in infants ${ }^{[35]}$. These effects are seen up to eight months post-partum, highlighting a longer-term impact on microbiota function. Affecting one in every seven live births worldwide ${ }^{[36]}$, gestational diabetes mellitus (GDM) presents the most frequent metabolic disorder that women encounter throughout gestation. The establishment of key pioneering taxa, important for establishing neonatal development, such as Lactobacillus, Flavonifractor, Erysipelotrichaceae and Bacteroides have shown to be decreased in infants born to mothers with GDM ${ }^{[37]}$. 


\section{Perinatal Factors Influencing Infant Gut Microbiome Establishment}

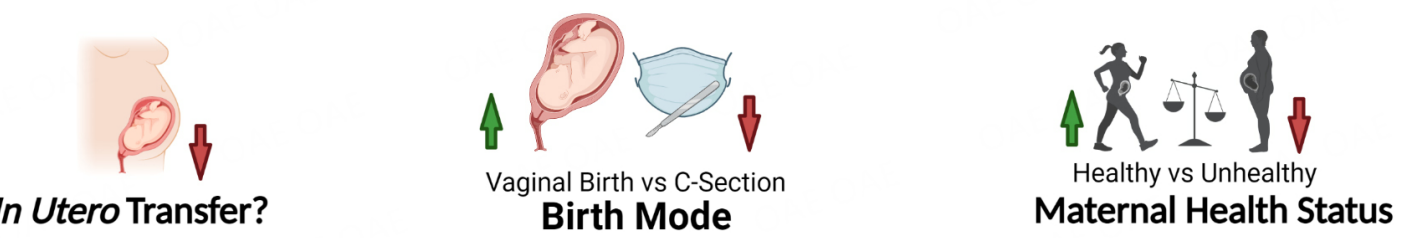

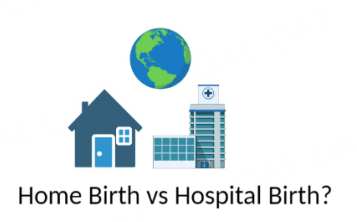

Environment

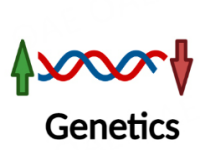

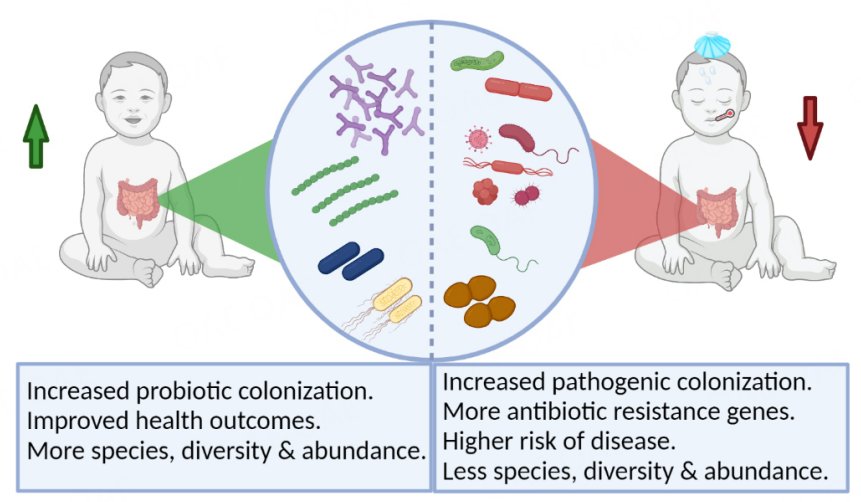

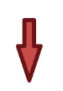

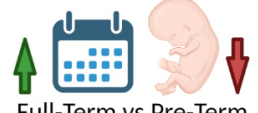

Full-Term vs Pre-Term

Gestational Age

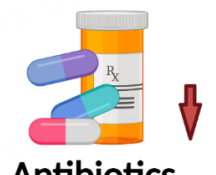

Antibiotics

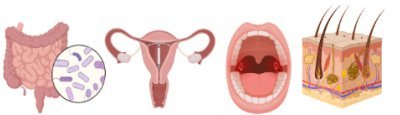

Gut, Vaginal, Oral \& Skin Microbiomes Mother-Infant Vertical Transmission

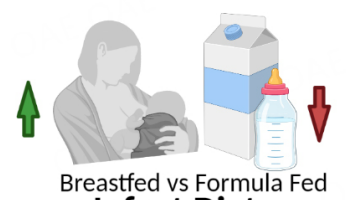

Infant Diet

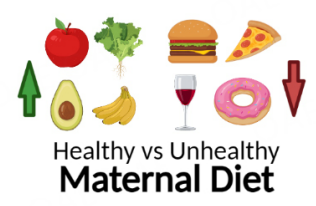

Figure 1. Perinatal factors influencing infant gut microbiome establishment. Graphic outlining the factors that contribute to early the initial infant gut microbiome. Created with BioRender.com.

Interestingly, the level of dysbiosis is postulated to occur differentially depending on the severity of the GDM condition ${ }^{[38]}$. Both extremes of the reproductive age (typically $<17$ years and $>35$ years) are considered as the highest risks for infant mortality and detrimental health outcomes for mother and infant. Fetal and maternal complications of GDM include fetal growth restriction, preterm birth, chromosomal and congenital abnormalities, pre-eclampsia and C-section (CS) birth ${ }^{[39-41]}$.

\section{Maternal diet}

Mounting evidence demonstrates the influence of a healthy maternal diet throughout the gestational period to optimize the acquisition of rich and diverse gut microbiota in neonates and improve long-term health outcomes $^{[42]}$. While the quintessential prenatal diet is not known, it is recommended that pregnant women consume "a balanced diet with the appropriate distribution of the basic food pyramid groups" ${ }^{\text {" } 43]}$. Regarding studies of the influence of maternal diet on infant gut microbiome establishment, it must be noted that notable heterogeneity pervades multiple areas of the studies presented to date. For instance, most studies utilize cohorts of overweight or obese women, which are not representative of a normal population. At present, there is no clear consensus on the periods of assessment and time points during pregnancy at which assessments are carried out. A systematic review by Maher et al. ${ }^{[42]}$ recently reported that inspection of detailed dietary data in pregnancy and its impact on the microbiome must be carried out in detail in a cohort that is indicative of a normal obstetric population. Bereft of this information, current findings from subgroups are challenging to decipher and highly variable. Finally, the authors noted that the methods of reporting dietary intake are subject to bias, suggesting that combining both Food Frequency Questionnaires and food diaries may be a method to circumvent this problem. With these limitations in mind, high-fat 
maternal diet has been repeatedly associated with detrimental effects on the infant gut microbiome ${ }^{[42]}$. Highfat maternal diet has been reported to be associated with the enrichment of Enterococcus and depletion in Bacteroides ${ }^{[4]}$. A recent study reported that diet in a healthy cohort of pregnant women was correlated to both maternal and neonatal microbiota at the time of birth, in a delivery mode-dependent manner ${ }^{[45]}$. Furthermore, the study indicated that fat intake in the form of saturated fatty acids and mono- and polyunsaturated fatty acids (MUFA and PUFA) showed significant enrichment in Firmicutes phylum genera and depletion in Proteobacteria phylum genera in the offspring's microbiota. A high-fat diet has been related to a higher abundance of Firmicutes and a concurrent increase in $\mathrm{BMI}^{[46,47]}$. Maternal intestinal permeability is higher throughout pregnancy ${ }^{[48]}$ and excess dietary intake is known to enhance intestinal permeability ${ }^{[49]}$, which may have implications on the vertical transmission of specific genera influencing early neonatal colonization. Prenatal diet has also been shown to modify the breast milk microbiome. High dietary intake of plant protein, fiber, and carbohydrates was associated with elevated Staphylococcus, Bifidobacterium, and Lactobacillus abundance ${ }^{[50]}$. The intake of food with high animal protein content and fats (n-3 PUFAs and MUFAs), exhibited a negative association with the abundance of Enterococcus and Bifidobacterium and a positive correlation with Gemella ${ }^{[50]}$ in the breast milk microbiome. Maternal diet may also modulate the human milk oligosaccharide (HMO) composition of breast milk ${ }^{[51]}$. Modulation of the HMO profile of breast milk by dietary manipulation may represent a method to manipulate the establishment of microbial species in the infant gut $^{[51]}$. Further studies are required to elucidate this relationship fully.

\section{In utero microbiome transfer}

Microbiome studies of the in utero environment highlight numerous limitations of current next-generation sequencing (NGS) methodologies. Contamination ${ }^{[52]}$ or the "kitome" ${ }^{[53]}$ represents a major issue when NGS and PCR-based approaches are applied to low-biomass samples, such as those encountered in the in utero environment ${ }^{[54]}$. Without stringent controls and method standardisation, these methodologies will amplify any DNA present in the sampled environment, regardless of bacterial viability ${ }^{[55]}$, thus, inaccurately reflecting the microbiota present in the sample ${ }^{[56]}$. Studies to date which have applied NGS methods informed by the potential for false-positive results have failed to detect a placental microbiome ${ }^{[53,57-60]}$. However, a well-controlled study by Edmond et al.${ }^{[6]]}$ indicated that the placenta represents a potential site of perinatal acquisition of Streptococcus agalactiae [group B Streptococcus (GBS)], a major cause of neonatal sepsis. Robust studies of amniotic fluid have failed to find a resident microbiome ${ }^{[62-64]}$. Indeed, Enterobacter and Escherichia, commonly identified contaminants in laboratory and extraction kit reagents ${ }^{[65,66]}$ have been reported as the most abundant genera detected in amniotic fluid ${ }^{[67,68]}$. Similarly, controlled studies have reported that the microbial content of meconium does not differ from negative control ${ }^{[6,70]}$. A recent study claimed to have detected bacterial DNA and viable bacteria in the fetal intestine using $16 \mathrm{~S}$ rRNA gene sequencing, qPCR, electron microscopy, and bacterial culture of Micrococcus luteus-related bacteria, which appeared to show adaptations to the fetal environment, re-igniting the controversy surrounding the existence of in utero microbiomes ${ }^{[7,72]}$. The studies presented thus far support the consensus that microbial colonization of the infant occurs at birth and the establishment of replicating microbial cells does not commonly occur in healthy pregnancies, devoid of clinical infections ${ }^{[73]}$. This is in agreement with Walter and Horneff ${ }^{[7]}$, who highlighted that there is no overlap between the bacterial taxa detected in utero in the sequencing studies of the in utero environment, with evident conformity between the bacterial taxa identified in utero and the controls.

\section{Birth mode}

Throughout vaginal birth, the infant is primarily exposed to the maternal intestinal and vaginal microbiota $^{[75]}$. Microbes of the birth canal such as Lactobacillus, Prevotella and Sneathia are typically detected in the infant gut of vaginally born infants. Bacteroides, Escherichia, Bifidobacterium, and 
Parabacteroides are also usually found ${ }^{[76]}$. These genera dominate the infant microbiota and comprise up to $68 \%$ of all microbes present four days after birth ${ }^{[77]}$. CS removes the infant's exposure to maternal intestinal and vaginal microbes, thus blocking vertical transmission and is the perinatal factor with the most uniform effects on the infant gut microbiota across individuals and studies ${ }^{[78]}$. A ubiquitous feature of the CS-born infant's gut microbiota is the low relative abundance of bifidobacteria and almost total lack of Bacteroides $^{[79]}$. The relative abundance of Enterococcus, Staphylococcus, Streptococcus, Klebsiella, Enterobacter, Propionibacterium, and Clostridium is also higher in the CS born infant gut ${ }^{[80]}$. Many of these taxa are common representatives of endemic opportunistic pathogens responsible for nosocomial infections ${ }^{[81]}$ and are common to the maternal skin and hospital environment ${ }^{[82]}$. The type of CS undertaken may further modulate its effects on the infant gut microbiome. For instance, the skin and vagina are thought to be the source of the gut microbiota in emergency CS, whereas the skin is considered to be the predominant microbial origin of the gut microbiota in infants born by elective $\mathrm{CS}^{[83]}$. Recent studies have attempted to utilise orally delivered fecal microbiota transplantation (FMT) and oral administration of maternal vaginal microbiota to restore disturbed intestinal microbial colonisation in CS born infants ${ }^{[8,85]}$. Despite its recent inception, FMT has yielded encouraging results ${ }^{[84]}$, while vaginal transplantation has delivered less comprehensive findings ${ }^{[85]}$.

\section{Mother-infant vertical transmission}

Following birth, infants appear to share $50 \%$ of microbial species in their gut with those found in the maternal gut, oral, vaginal, or skin microbiota indicating vertical transmission of microbes from mother to infant ${ }^{[86]}$. A study by Ferretti et al. ${ }^{[86]}$ reported that a significant proportion $(50.7 \%)$ of the microbes present in the infant gut immediately post-partum, were transferred from the mother's gut, vagina, oral cavity, or skin. The presence of these microbes was largely stable up to four months of age ${ }^{[86]}$. The mother's gut accounted for the most significant proportion of microbes (22.1\%), followed by the vagina (16.3\%), the oral cavity $(7.2 \%)$, and the skin $(5 \%)^{[86]}$. The maternal gut microbiota represents the greatest maternal source of infant-acquired strains immediately post-partum, with most strains from Actinobacteria and Bacteroidetes $^{[16]}$. Furthermore, a recent large scale meta-analysis of whole metagenomic shotgun sequencing data of maternal and infant fecal samples identified a set of 26 mother-infant shared species (species of $B$. uniformis, B. vulgatus, B. longum, $P$. distasonis, $P$. merdae confirmed at strain level) with high prevalence and relative abundance across cohorts studied ${ }^{[87]}$. The vaginal microbiome during gestation is dominated by Lactobacillus species, displaying decreased diversity and increased stability compared to non-pregnant women $^{[88]}$. The vaginally delivered infant gut microbiome is dominated by Lactobacillus, Prevotella, Atopobium or Sneathia species, which are typical of the mother's vaginal microbiome ${ }^{[89]}$ The vaginal microbiomes impact on the infant before birth remains largely unknown, however gestational changes that happen during pregnancy could be part of an adaptive response to promote the correct development and health of the fetus ${ }^{[0]}$. While the vaginal microbiome does contribute bacteria to the infant gut microbiome it may be that this contribution is minimal, in terms of overall abundance due to the low diversity of the vaginal microbiome during pregnancy ${ }^{[8]}$. The mother's oral cavity and skin represent the other possible maternal sources of microbes. The contributions of microbes from these areas to the initial infant gut microbiome are significantly less than the mothers gut, vaginal and breast milk microbiomes. Furthermore, bacteria transferred from these sites colonize the infant gut in a transient manner ${ }^{[91,92]}$. However, significant microbial transfer from the mother's skin and oral cavity to infants in later life is feasible, although additional studies are needed.

\section{Gestational age}

Gestational age is one of the most significant influencers of gut microbiota formation ${ }^{[93]}$, defined as full-term (37-42 weeks), preterm (<37 weeks) or post-term ( $>42$ weeks). The intestinal microbiota of the preterm infant is distinguished by delayed colonization, diminished species diversity and abundance. There is also a 
predilection to being colonized by pathogenic facultative anaerobes (e.g., Enterobacter, Escherichia, and Klebsiella) and decreased levels of commensal strictly anaerobic organisms (e.g., Bifidobacterium, Bacteroides, and Clostridium $)^{[94]}$. Numerous studies have indicated a shared patterned progression of colonization by bacilli, Gammaproteobacteria and Clostridia in preterm infants ${ }^{[95]}$. In particular, Enterococcus faecalis, Enterobacter cloacae, Staphylococcus epidermidis, E. coli, Klebsiella pneumoniae, and Klebsiella oxytoca species are frequently found in the gut microbiota of preterm infants ${ }^{[96]}$. Preterm infants also encounter numerous distinctive environmental and host conditions, which can have deleterious effects on the establishment and subsequent formation of their gut microbiome. For instance, even before birth, between $25 \%-30 \%$ of preterm infants are exposed to microbes in the context of preterm premature rupture of membranes and intra-amniotic infection ${ }^{[97]}$. CS birth is also common in preterm birth cases; thus, colonization by the skin and environmental conditions rather than vaginal and rectal microbiota commonly occur $^{[78]}$. Furthermore, exposure to the Neonatal Intensive Care Unit (NICU) has been related with a NICUassociated core microbiota composed of bacterial families Enterobacteriaceae (genera Klebsiella and Escherichia in particular) and Enterococcaceae ${ }^{[98]}$. A common NICU practice, respiratory support, has recently been shown to drive differences in microbiota development between extremely and very preterm infants ${ }^{[8]}$. Specifically, preterm infants exhibited higher colonization by facultative anaerobes and delayed colonization by obligate anaerobes ${ }^{[98]}$. As a result, with the shift in the ratio of facultative to obligate anaerobic bacteria, defense against pathogenic bacteria can often be impaired ${ }^{\left[{ }^{[3]}\right]}$. Finally, antibiotic administration in NICUs is commonplace for treating and preventing infections and sepsis ${ }^{[93]}$ and will be discussed in detail below.

\section{Antibiotics}

In light of the ever-evolving antimicrobial resistance (AMR) crisis ${ }^{[99]}$, deciphering the implications of perinatal antibiotic usage on the establishment of the infant gut microbiome is paramount. Oral antibiotic use in pregnancy or intravenous antibiotic prophylaxis during delivery is common practice - especially for maternal GBS positivity, preterm premature rupture of the membranes, and/or as prophylaxis against wound infection in $\mathrm{CS}^{[100]}$. Vertical transmission of antibiotics and antibiotic-resistant microorganisms from mother to infant has been proposed to negatively impact the development and succession of the infant gut microbiota $^{[101]}$. Antibiotic usage presents two potential problems in the perinatal period, through the transfer of antibiotics and/or AMR strains from mother to infant via in utero transfer or breastfeeding. The majority of broad-spectrum antibiotics (e.g., amoxicillin, gentamicin, vancomycin) used in the perinatal period can be readily transmitted to the fetus in utero via the placenta and umbilical vein due to simple diffusion and blood flow ${ }^{[102]}$. Because of the restricted activity of fetal hepatic drug-metabolizing enzymes compared with adults, the non-metabolized drug thus accumulates in the fetal tissues ${ }^{[103]}$. There is strong evidence that maternal antibiotic treatment throughout gestation reaches the infant in adequate amounts to potentially impact their resistome profiles ${ }^{[104]}$. Numerous studies have explored the effect of antibiotic use in pregnancy on infants' intestinal microbiome composition and/or diversity. Reduced relative abundances of Actinobacteria, specifically Bifidobacterium ${ }^{[104]}$ and Bacteroides ${ }^{[105]}$ are commonly observed in infants whose mothers were treated with antibiotics prenatally or during delivery compared to those without. Increased relative abundances of Firmicutes and Proteobacteria are also consistently reported ${ }^{[106]}$. To date, most studies concentrate on the detrimental effects of antibiotics on the mother or fetus but lack exploration of the AMR aspects. There is also a pressing need for characterization of the species and strains harbouring these resistance genes ${ }^{[107]}$.

\section{Infant diet}

Human breast milk is the "gold standard" nutrition for infant health and development and is considered the second integral source of microbes to the infant after the birth canal ${ }^{[108]}$. Harbouring $>700$ bacterial species, breast milk ensures the consumption of up to $\sim 800,000$ bacteria daily ${ }^{[109]}$. HMOs modulate the immune 
system, prevent adhesion of pathogenic bacteria and act as metabolic substrates for gut bifidobacteria. In turn, bifidobacteria utilize their arsenal of membrane transporters and saccharolytic enzymes to cleave HMOs into their constituent monomers and internalize these or intact HMOs into the central catabolic pathways, leading to the main end products, lactate, acetate, formate, and 1, 2-propandiol ${ }^{[110]}$. This process aids in suppressing the growth of opportunistic pathogenic species within Clostridiaceae, Enterobacteriaceae, and Staphylococcaceae ${ }^{[111]}$. Multiple studies have isolated and identified bacterial strains, mostly Bifidobacterium, Lactobacillus, and Staphylococcus, which are common to both breast milk and infant faeces ${ }^{[112]}$. However, breastfeeding is not chosen/not possible in many circumstances. Exclusively formula-fed infants harbor a more diverse microbiota with lower abundances of HMO-utilizing Bifidobacterium species, often with increased abundances of Clostridium species (C. difficile and C. perfringens) and Enterobacteriaceae species (e.g., E. coli) ${ }^{[113]}$. While the gap between the composition of commercial formulas and breast milk is narrowing ${ }^{[114]}$, the gut microbiota of formula and breast-fed infants remain distinct ${ }^{[35]}$.

\section{Probiotics}

Probiotics are defined by the FAO/WHO as "live microorganisms which when administered in adequate amounts confer a health benefit on the host" ${ }^{\text {[15] }}$. Probiotic supplementation in infants to ameliorate aberrant colonization, typically with Bifidobacterium and Lactobacillus strains, has yielded mixed results ${ }^{[10]}$. Probiotic usage for the prevention of NEC in preterm infants has yielded encouraging finding $\mathrm{s}^{[116]}$. A recent metaanalysis concluded that $L$. acidophilus LB was the best supplementation option for reducing NEC risk in preterm infants ${ }^{[17]} \mathrm{A}$ synbiotic is defined as "a mixture comprising live microorganisms and substrate(s) selectively utilized by host microorganisms that confers a health benefit on the host" ${ }^{\text {"[18] }}$. A synbiotic consisting of short-chain galacto-oligosaccharides and long-chain fructo-oligosaccharides and the bifidobacterial strain $B$. breve $\mathrm{M}-16 \mathrm{~V}$, with a similar dosage to bacterial numbers of human milk, was reported to substantially increase levels of bifidobacteria in exclusively formula-fed infants ${ }^{[119]}$. Further studies of probiotic, prebiotic and synbiotic supplementation in the infant diet to ensure optimal microbial colonization of the infant gut are warranted.

\section{Genetics}

Individuals with different genetic backgrounds harbour distinct microbes, as have been indicated by host and microbiome genome-wide association studies. These studies show similarities in the gut microbiome composition among phylogenetically-related individuals ${ }^{[120]}$. For instance, twin studies indicate that the microbiome of monozygotic twins is significantly closer than that of dizygotic twins ${ }^{[122]}$. Kurilshikov et al. ${ }^{[122]}$ reported an age-dependent association between the lactase (LCT) gene locus and the Bifidobacterium genus in the MiBioGen consortium. Indeed, the functional SNP in the LCT locus, rs4988235, has been shown to determine the abundance of the Bifidobacterium genus ${ }^{[123]}$. The DR4-DQ8 allele, the highest risk associated allele for type 1 diabetes, has also been hypothesised to serve as a gatekeeper for the presence or absence of gut bacteria in early life ${ }^{[124]}$. A study of dichorionic triplets suggested that host genetics initially play a major role in determining microbial community composition; however, by year one, environmental factors are the major determinant in healthy infants ${ }^{[125]}$, suggesting that nurture has the potential to overcome the genetic nature of an individual. Collectively, the findings of these studies demonstrated the influence of host genetics in the formation and development of the human microbiota. Further studies are needed to fully elucidate how genetic differences may shape the mother-to-infant microbiota transmission and the formation of the gut microbiome in the initial stages of life.

\section{Environment}

Environmental factors such as place of birth and geographical location have also been reported to influence initial patterns of infant gut microbiota colonization. For instance, while the hospital setting contributes an 
antiseptic environment for labor and delivery, it also presents a possible exposure route of antibioticresistant bacteria, especially in the case of CS birth ${ }^{[82]}$. Lower beta diversity of Bacteroides, Bifidobacterium, Streptococcus and Lactobacillus and higher Clostridium and Enterobacteriaceae in hospital-born infants than babies delivered at home has been demonstrated ${ }^{[126]}$. Similarly, a study indicated that the microbiota of hospital-delivered infants was enriched with gram-positive anaerobic cocci, including the Peptoniphilus and Finegoldia genera, while those of home-delivered infants exhibited higher relative abundances of species from the Enterococcus and Bifidobacterium genera $^{[127]}$. Geographical location and ethnicity are also proposed to impact the establishment of the infant gut microbiome; however, these microbiota differences seem to be associated with dietary and lifestyle patterns in a specific area or region ${ }^{[128]}$. For instance, children living in a rural village in Africa harbor a distinct microbiota to children residing in an urban region in Italy ${ }^{[129]}$. African infants show an increased relative abundance of bacilli, while North American infants have more Bacteroidetes and less Actinobacteria than infants in other continents ${ }^{[79]}$. Numerous additional studies have explored the geographical effect, as linked to ethnicity and/or diet, on microbial diversity and composition $^{[130]}$.

\section{CONCLUSION}

Evidence to date indicates that perinatal factors have a significant effect on the formation of the gut microbiota in the initial stages of life. Our understanding of exactly how such factors affect microbial colonization and, in turn, influence infant health is mostly limited to correlation rather than causation studies due to a previously underpowered toolkit. Furthermore, direct comparisons of studies of infant gut microbiota and perinatal factors have been limited due to the cultivation techniques used and the use of $16 \mathrm{~S}$ rRNA amplicon sequencing, which have informed most of our understanding of the human microbiome to date. Yet, these approaches are hampered by the limited number of cultivable species ${ }^{[131]}$, primer design ${ }^{[132]}$, sample processing ${ }^{[133]}$, small sample sizes ${ }^{[113]}$ and the low taxonomic resolution provided by $16 \mathrm{~S}$ rRNA gene profiling ${ }^{[134]}$. Most importantly, as $16 \mathrm{~S}$ rRNA amplicon sequencing is only reliable to genus level, it does not provide information about the functional capacity of the microbiome. Fortunately, the advent of metagenomics sequencing (i.e., whole-genome shotgun sequencing) has illuminated much of the "dark matter" of our microbiome ${ }^{[135]}$ and will hopefully circumvent many of the limitations of previous studies. These methods allow characterization of microbial activity, helping us to elucidate not only which microbes are present, but also what metabolic functionalities these microbes possess. Such knowledge could uncover associations between the presence of particular microbes and particular neonatal diseases.

A deeper understanding of how perinatal factors may stimulate or hinder the inheritance and/or choice of microbes by infants in early life constitutes a viable strategy in the investigation of next-generation probiotics for therapeutic interventions that maintain/improve the health of infants. Large scale longitudinal human studies, with greater, equally balanced cohorts given the same strains and prescription of either prebiotics, probiotics or synbiotics at comparable times and by related delivery routes are needed to target the vertical transmission of maternal microbes to infants with regards to specific bacteria at higher taxonomic resolution (e.g., strains) and the gross changes of the infant's microbiota.

Studies investigating how multiple perinatal factors may interact with each other in the formation of the infant gut microbiome are also warranted. For instance, breastfeeding has been shown to beneficially alter the patterns of aberrant colonisation in CS born infants ${ }^{[136]}$. Finally, detailed transcriptomics, metabolomics, lipidomics and proteomic investigations in combination with DNA and RNA sequencing will enable us to fully uncover the effects of perinatal factors on initial microbiome establishment and long-term functioning. 


\section{DECLARATIONS}

\section{Authors' contributions}

Conceptualization: Stanton C, Dempsey EM, Ryan CA, Ross RP

Writing - original draft preparation: Linehan $\mathrm{K}$

Edited the manuscript: Linehan K, Dempsey EM, Ryan CA, Ross RP, Stanton C

\section{Availability of data and materials}

Not applicable.

\section{Financial support and sponsorship}

This research is supported by APC Microbiome Ireland, a research centre funded by Science Foundation Ireland (SFI), through the Irish Government's National Development Plan (Grant Number. 12/RC/2273_P2).

\section{Conflicts of interest}

All authors declared that there are no conflicts of interest.

\section{Ethical approval and consent to participate}

Not applicable.

\section{Consent for publication}

Not applicable.

\section{Copyright}

(C) The Author(s) 2022.

\section{REFERENCES}

1. Berg G, Rybakova D, Fischer D, et al. Microbiome definition re-visited: old concepts and new challenges. Microbiome 2020;8:103. DOI PubMed PMC

2. Lloyd-Price J, Abu-Ali G, Huttenhower C. The healthy human microbiome. Genome Medicine 2016;8:51. DOI PubMed PMC

3. Gensollen T, Iyer SS, Kasper DL, Blumberg RS. How colonization by microbiota in early life shapes the immune system. Science 2016;352:539-44. DOI PubMed PMC

4. Nguyen QP, Karagas MR, Madan JC, et al. Associations between the gut microbiome and metabolome in early life. BMC Microbiol 2021;21:1-19. DOI PubMed PMC

5. Fan Y, Pedersen O. Gut microbiota in human metabolic health and disease. Nat Rev Microbiol 2021;19:55-71. DOI PubMed

6. Gopalakrishnan V, Helmink BA, Spencer CN, Reuben A, Wargo JA. The influence of the gut microbiome on cancer, immunity, and cancer immunotherapy. Cancer Cell 2018;33:570-80. DOI PubMed PMC

7. Murphy K, O’Donovan AN, Caplice NM, Ross RP, Stanton C. Exploring the gut microbiota and cardiovascular disease. Metabolites 2021;11:493. DOI PubMed PMC

8. Clarke G, O’Mahony S, Dinan T, Cryan J. Priming for health: gut microbiota acquired in early life regulates physiology, brain and behaviour. Acta Paediatr 2014;103:812-9. DOI PubMed

9. Fouhy F, Watkins C, Hill CJ, et al. Perinatal factors affect the gut microbiota up to four years after birth. Nat Commun $2019 ; 10: 1517$. DOI PubMed PMC

10. Wang S, Egan M, Ryan CA, et al. A good start in life is important-perinatal factors dictate early microbiota development and longer term maturation. FEMS Microbiol Rev 2020;44:763-81. DOI PubMed PMC

11. Fundora JB, Guha P, Shores DR, Pammi M, Maheshwari A. Intestinal dysbiosis and necrotizing enterocolitis: assessment for causality using Bradford Hill criteria. Pediatr Res 2020;87:235-48. DOI PubMed PMC

12. Milani C, Duranti S, Bottacini F, et al. The first microbial colonizers of the human gut: composition, activities, and health implications of the infant gut microbiota. Microbiol Mol Biol Rev 2017;81:e00036-17. DOI PubMed PMC

13. Costello EK, Stagaman K, Dethlefsen L, Bohannan BJM, Relman DA. The application of ecological theory toward an understanding of the human microbiome. Science 2012;336:1255-62. DOI PubMed PMC

14. Arrieta MC, Stiemsma LT, Amenyogbe N, Brown EM, Finlay B. The intestinal microbiome in early life: health and disease. Front Immunol 2014;5:427. DOI PubMed PMC

15. Collado MC, Cernada M, Baüerl C, Vento M, Pérez-Martínez G. Microbial ecology and host-microbiota interactions during early life stages. Gut Microbes 2012;3:352-65. DOI PubMed PMC 
16. Duranti S, Lugli GA, Mancabelli L, et al. Maternal inheritance of bifidobacterial communities and bifidophages in infants through vertical transmission. Microbiome 2017;5:66. DOI PubMed PMC

17. Turroni F, Milani C, Duranti S, et al. The infant gut microbiome as a microbial organ influencing host well-being. Ital J Pediatr 2020;46:16. DOI PubMed PMC

18. Alessandri G, van Sinderen D, Ventura M. The genus bifidobacterium: from genomics to functionality of an important component of the mammalian gut microbiota running title: Bifidobacterial adaptation to and interaction with the host. Comput Struct Biotechnol J 2021;19:1472-87. DOI PubMed PMC

19. Wiley NC, Cryan JF, Dinan TG, Ross RP, Stanton C. Production of psychoactive metabolites by gut bacteria. Mod Trends Psychiatry 2021;32:74-99. DOI PubMed

20. Yoshii K, Hosomi K, Sawane K, Kunisawa J. Metabolism of dietary and microbial vitamin B family in the regulation of host immunity. Front Nutr 2019;6:48. DOI PubMed PMC

21. Marcobal A, Barboza M, Sonnenburg ED, et al. Bacteroides in the infant gut consume milk oligosaccharides via mucus-utilization pathways. Cell Host Microbe 2011;10:507-14. DOI PubMed PMC

22. Otaru N, Ye K, Mujezinovic D, et al. GABA production by human intestinal Bacteroides spp.: prevalence, regulation, and role in acid stress tolerance. Front Microbiol 2021;12:656895. DOI PubMed PMC

23. Rios-Covian D, Salazar N, Gueimonde M, de Los Reyes-Gavilan CG. Shaping the metabolism of intestinal Bacteroides population through diet to improve human health. Front Microbiol 2017;8:376. DOI PubMed PMC

24. Vipperla K, O'Keefe SJ. The microbiota and its metabolites in colonic mucosal health and cancer risk. Nutr Clin Pract 2012;27:62435. DOI PubMed

25. Han KI, Kim JS, Eom MK, et al. Collinsella acetigenes sp. nov., an Anaerobic Actinobacterium Isolated from Human Feces, and Emended Description of the Genus Collinsella and Collinsella aerofaciens. Curr Microbiol 2021;78:3667-73. DOI PubMed

26. Magnúsdóttir S, Ravcheev D, de Crécy-Lagard V, Thiele I. Systematic genome assessment of B-vitamin biosynthesis suggests cooperation among gut microbes. Front Genet 2015;6:148. DOI PubMed PMC

27. Zhang X, Mushajiang S, Luo B, et al. The composition and concordance of Lactobacillus populations of infant gut and the corresponding breast-milk and maternal gut. Front Microbiol 2020;11:597911. DOI PubMed PMC

28. Yaghoubfar R, Behrouzi A, Ashrafian F, et al. Modulation of serotonin signaling/metabolism by Akkermansia muciniphila and its extracellular vesicles through the gut-brain axis in mice. Sci Rep 2020;10:22119. DOI PubMed PMC

29. Mancabelli L, Tarracchini C, Milani C, et al. Multi-population cohort meta-analysis of human intestinal microbiota in early life reveals the existence of infant community state types (ICSTs). Comput Struct Biotechnol J 2020;18:2480-93. DOI PubMed PMC

30. Taboada B, Morán P, Serrano-Vázquez A, et al. The gut virome of healthy children during the first year of life is diverse and dynamic. PLoS One 2021;16:e240958. DOI PubMed PMC

31. Egan M, Dempsey E, Ryan CA, Ross RP, Stanton C. The Sporobiota of the human gut. Gut Microbes 2021;13:1-17. DOI PubMed PMC

32. Naudé PJW, Claassen-Weitz S, Gardner-Lubbe S, et al. Association of maternal prenatal psychological stressors and distress with maternal and early infant faecal bacterial profile. Acta Neuropsychiatr 2020;32:32-42. DOI PubMed PMC

33. National Research C. Weight gain during pregnancy: reexamining the guidelines. Washington (DC): National Academies Press (US); 2009. DOI PubMed

34. Singh SB, Madan J, Coker M, et al. Does birth mode modify associations of maternal pre-pregnancy BMI and gestational weight gain with the infant gut microbiome? Int J Obes (Lond) 2020;44:23-32. DOI PubMed PMC

35. Baumann-Dudenhoeffer AM, D'Souza AW, Tarr PI, Warner BB, Dantas G. Infant diet and maternal gestational weight gain predict early metabolic maturation of gut microbiomes. Nat Med 2018;24:1822-9. DOI PubMed PMC

36. Cho NH, Shaw JE, Karuranga S, et al. IDF Diabetes Atlas: global estimates of diabetes prevalence for 2017 and projections for 2045. Diabetes Res Clin Pract 2018;138:271-81. DOI PubMed

37. Soderborg TK, Carpenter CM, Janssen RC, et al. Gestational diabetes is uniquely associated with altered early seeding of the infant gut microbiota. Front Endocrinol (Lausanne) 2020;11:603021. DOI PubMed PMC

38. Sililas P, Huang L, Thonusin C, et al. Association between gut microbiota and development of gestational diabetes mellitus. Microorganisms 2021;9:1686. DOI PubMed PMC

39. Kim YN, Choi DW, Kim DS, Park EC, Kwon JY. Maternal age and risk of early neonatal mortality: a national cohort study. Sci Rep 2021;11:814. DOI PubMed PMC

40. Frick AP. Advanced maternal age and adverse pregnancy outcomes. Best Pract Res Clin Obstet Gynaecol 2021;70:92-100. DOI PubMed

41. Attali E, Yogev Y. The impact of advanced maternal age on pregnancy outcome. Best Pract Res Clin Obstet Gynaecol 2021;70:2-9. DOI PubMed

42. Maher SE, O'Brien EC, Moore RL, et al. The association between the maternal diet and the maternal and infant gut microbiome: a systematic review. Br J Nutr 2020:1-29. DOI PubMed

43. Society for Reproductive Medicine, American College of Obstetricians and Gynecologists' Committee on Gynecologic Practice. Prepregnancy counseling: Committee Opinion No. 762. Fertil Steril 2019;111:32-42. DOI PubMed

44. Chu DM, Antony KM, Ma J, et al. The early infant gut microbiome varies in association with a maternal high-fat diet. Genome Med 2016;8:77. DOI PubMed PMC

45. Selma-Royo M, García-Mantrana I, Calatayud M, Parra-Llorca A, Martínez-Costa C, Collado MC. Maternal diet during pregnancy and intestinal markers are associated with early gut microbiota. Eur J Nutr 2021;60:1429-42. DOI PubMed 
46. Koliada A, Syzenko G, Moseiko V, et al. Association between body mass index and Firmicutes/Bacteroidetes ratio in an adult Ukrainian population. BMC Microbiol 2017;17:120. DOI PubMed PMC

47. Serino M, Luche E, Gres S, et al. Metabolic adaptation to a high-fat diet is associated with a change in the gut microbiota. Gut 2012;61:543-53. DOI PubMed PMC

48. Reyes H, Zapata R, Hernández I, et al. Is a leaky gut involved in the pathogenesis of intrahepatic cholestasis of pregnancy? Hepatology 2006;43:715-22. DOI PubMed

49. Rohr MW, Narasimhulu CA, Rudeski-Rohr TA, Parthasarathy S. Negative effects of a high-fat diet on intestinal permeability: a review. Adv Nutr 2020;11:77-91. DOI PubMed PMC

50. Cortes-Macías E, Selma-Royo M, García-Mantrana I, et al. Maternal diet shapes the breast milk microbiota composition and diversity: impact of mode of delivery and antibiotic exposure. J Nutr 2021;151:330-40. DOI PubMed PMC

51. Seferovic MD, Mohammad M, Pace RM, et al. Maternal diet alters human milk oligosaccharide composition with implications for the milk metagenome. Sci Rep 2020;10:22092. DOI PubMed PMC

52. de Goffau MC, Lager S, Salter SJ, et al. Recognizing the reagent microbiome. Nat Microbiol 2018;3:851-3. DOI PubMed

53. Olomu IN, Pena-Cortes LC, Long RA, et al. Elimination of "kitome" and "splashome" contamination results in lack of detection of a unique placental microbiome. BMC Microbiol 2020;20:157. DOI PubMed PMC

54. Bushman FD. De-discovery of the placenta microbiome. Am J Obstet Gynecol 2019;220:213-4. DOI PubMed

55. Amos GCA, Logan A, Anwar S, et al. Developing standards for the microbiome field. Microbiome 2020;8:98. DOI PubMed PMC

56. Fricke WF, Ravel J. Microbiome or no microbiome: are we looking at the prenatal environment through the right lens? Microbiome 2021;9:9. DOI PubMed PMC

57. Lauder AP, Roche AM, Sherrill-Mix S, et al. Comparison of placenta samples with contamination controls does not provide evidence for a distinct placenta microbiota. Microbiome 2016;4:29. DOI

58. Leiby JS, McCormick K, Sherrill-Mix S, et al. Lack of detection of a human placenta microbiome in samples from preterm and term deliveries. Microbiome 2018;6:196. DOI PubMed PMC

59. Theis KR, Romero R, Winters AD, et al. Does the human placenta delivered at term have a microbiota? Am J Obstet Gynecol 2019;220:267.e1-267.e39. DOI PubMed PMC

60. Leon LJ, Doyle R, Diez-Benavente E, et al. Enrichment of clinically relevant organisms in spontaneous preterm-delivered placentas and reagent contamination across all clinical groups in a large pregnancy cohort in the United Kingdom. Appl Environ Microbiol 2018;84:e0483-18. DOI PubMed PMC

61. Edmond KM, Kortsalioudaki C, Scott S, et al. Group B streptococcal disease in infants aged younger than 3 months: systematic review and meta-analysis. Lancet 2012;379:547-56. DOI PubMed

62. Lim ES, Rodriguez C, Holtz LR. Amniotic fluid from healthy term pregnancies does not harbor a detectable microbial community. Microbiome 2018;6:87. DOI PMC

63. Rehbinder EM, Lødrup Carlsen KC, Staff AC, et al. Is amniotic fluid of women with uncomplicated term pregnancies free of bacteria? Am J Obstet Gynecol 2018;219:289.e1-289.e12. DOI PubMed

64. de Goffau MC, Lager S, Sovio U, et al. Human placenta has no microbiome but can contain potential pathogens. Nature 2019;572:329-34. DOI PubMed PMC

65. Salter SJ, Cox MJ, Turek EM, et al. Reagent and laboratory contamination can critically impact sequence-based microbiome analyses. BMC Biol 2014;12:87. DOI PubMed PMC

66. Tanner MA, Goebel BM, Dojka MA, Pace NR. Specific ribosomal DNA sequences from diverse environmental settings correlate with experimental contaminants. Appl Environ Microbiol 1998;64:3110-3. DOI PubMed PMC

67. Liu CJ, Liang X, Niu ZY, et al. Is the delivery mode a critical factor for the microbial communities in the meconium? EBioMedicine 2019;49:354-63. DOI PubMed PMC

68. Collado MC, Rautava S, Aakko J, Isolauri E, Salminen S. Human gut colonisation may be initiated in utero by distinct microbial communities in the placenta and amniotic fluid. Sci Rep 2016;6:23129. DOI PubMed PMC

69. Kennedy KM, Gerlach MJ, Adam T, et al. Fetal meconium does not have a detectable microbiota before birth. Nat Microbiol 2021;6:865-73. DOI PubMed

70. Santos SJ, Pakzad Z, Elwood CN, et al; Maternal Microbiome Legacy Project Team. Early neonatal meconium does not have a demonstrable microbiota determined through use of robust negative controls with cpn60-based microbiome profiling. Microbiol Spectr 2021;9:e006721. DOI PubMed PMC

71. Rackaityte E, Halkias J, Fukui EM, et al. Corroborating evidence refutes batch effect as explanation for fetal bacteria. Microbiome 2021;9:10. DOI PubMed PMC

72. Goffau MC, Charnock-Jones DS, Smith GCS, Parkhill J. Batch effects account for the main findings of an in utero human intestinal bacterial colonization study. Microbiome 2021;9:6. DOI

73. Blaser MJ, Devkota S, McCoy KD, Relman DA, Yassour M, Young VB. Lessons learned from the prenatal microbiome controversy. Microbiome 2021;9:8. DOI PubMed PMC

74. Walter J, Hornef MW. A philosophical perspective on the prenatal in utero microbiome debate. Microbiome 2021;9:5. DOI PubMed PMC

75. Li N, Liang S, Chen Q, Zhao L, Li B, Huo G. Distinct gut microbiota and metabolite profiles induced by delivery mode in healthy Chinese infants. J Proteomics 2021;232:104071. DOI PubMed

76. Wampach L, Heintz-Buschart A, Fritz JV, et al. Birth mode is associated with earliest strain-conferred gut microbiome functions and immunostimulatory potential. Nat Commun 2018;9:5091. DOI PubMed PMC 
77. Shao Y, Forster SC, Tsaliki E, et al. Stunted microbiota and opportunistic pathogen colonization in caesarean-section birth. Nature 2019;574:117-21. DOI PubMed PMC

78. Korpela K. Impact of delivery mode on infant gut microbiota. Ann Nutr Metab 2021:1-9. DOI PubMed

79. Korpela K, de Vos WM. Early life colonization of the human gut: microbes matter everywhere. Curr Opin Microbiol 2018;44:70-8. DOI PubMed

80. Shaterian N, Abdi F, Ghavidel N, Alidost F. Role of cesarean section in the development of neonatal gut microbiota: a systematic review. Open Med (Wars) 2021;16:624-39. DOI PubMed PMC

81. O'Neill IJ, Sanchez Gallardo R, Saldova R, et al. Maternal and infant factors that shape neonatal gut colonization by bacteria. Expert Rev Gastroenterol Hepatol 2020;14:651-64.10. DOI PubMed

82. Lax S, Sangwan N, Smith D, et al. Bacterial colonization and succession in a newly opened hospital. Sci Transl Med 2017;9:eaah6500. DOI PubMed PMC

83. Chu DM, Ma J, Prince AL, Antony KM, Seferovic MD, Aagaard KM. Maturation of the infant microbiome community structure and function across multiple body sites and in relation to mode of delivery. Nat Med 2017;23:314-26. DOI PubMed PMC

84. Korpela K, Helve O, Kolho KL, et al. Maternal fecal microbiota transplantation in cesarean-born infants rapidly restores normal gut microbial development: a proof-of-concept study. Cell 2020;183:324-34.e5. DOI PubMed

85. Wilson BC, Butler ÉM, Grigg CP, et al. Oral administration of maternal vaginal microbes at birth to restore gut microbiome development in infants born by caesarean section: a pilot randomised placebo-controlled trial. EBioMedicine 2021;69:103443. DOI PubMed PMC

86. Ferretti P, Pasolli E, Tett A, et al. Mother-to-infant microbial transmission from different body sites shapes the developing infant gut microbiome. Cell Host Microbe 2018;24:133-45.e5. DOI PubMed PMC

87. Wang S, Zeng S, Egan M, et al. Metagenomic analysis of mother-infant gut microbiome reveals global distinct and shared microbial signatures. Gut Microbes 2021;13:1-24. DOI PubMed PMC

88. Mortensen MS, Rasmussen MA, Stokholm J, et al. Modeling transfer of vaginal microbiota from mother to infant in early life. Elife 2021;10:e57051. DOI PubMed PMC

89. Chen X, Lu Y, Chen T, Li R. The female vaginal microbiome in health and bacterial vaginosis. Front Cell Infect Microbiol 2021;11:631972. DOI PubMed PMC

90. Mueller NT, Bakacs E, Combellick J, Grigoryan Z, Dominguez-Bello MG. The infant microbiome development: mom matters. Trends Mol Med 2015;21:109-17. DOI PubMed PMC

91. Dominguez-Bello MG, Costello EK, Contreras M, et al. Delivery mode shapes the acquisition and structure of the initial microbiota across multiple body habitats in newborns. Proc Natl Acad Sci U S A 2010;107:11971-5. DOI PubMed PMC

92. Hurley E, Mullins D, Barrett MP, et al. The microbiota of the mother at birth and its influence on the emerging infant oral microbiota from birth to 1 year of age: a cohort study. J Oral Microbiol 2019;11:1599652. DOI PubMed PMC

93. Henderickx JGE, Zwittink RD, van Lingen RA, Knol J, Belzer C. The preterm gut microbiota: an inconspicuous challenge in nutritional neonatal care. Front Cell Infect Microbiol 2019;9:85. DOI PubMed PMC

94. Pammi M, Cope J, Tarr PI, et al. Intestinal dysbiosis in preterm infants preceding necrotizing enterocolitis: a systematic review and meta-analysis. Microbiome 2017;5:31. DOI PubMed PMC

95. La Rosa PS, Warner BB, Zhou Y, et al. Patterned progression of bacterial populations in the premature infant gut. Proc Natl Acad Sci U S A 2014;111:12522-7. DOI PubMed PMC

96. Nguyen M, Holdbrooks H, Mishra P, et al. Impact of probiotic B. infantis EVC001 feeding in premature infants on the gut microbiome, nosocomially acquired antibiotic resistance, and enteric inflammation. Front Pediatr 2021;9:618009. DOI PubMed PMC

97. Goldenberg RL, Culhane JF, Iams JD, Romero R. Epidemiology and causes of preterm birth. Lancet 2008;371:75-84. DOI PubMed PMC

98. Zwittink RD, van Zoeren-Grobben D, Martin R, et al. Metaproteomics reveals functional differences in intestinal microbiota development of preterm infants. Mol Cell Proteomics 2017;16:1610-20. DOI PubMed PMC

99. Ventola CL. The antibiotic resistance crisis: part 1: causes and threats. P T 2015;40:277-83. PubMed PMC

100. Grech A, Collins CE, Holmes A, et al. Maternal exposures and the infant gut microbiome: a systematic review with meta-analysis. Gut Microbes 2021;13:1-30. DOI PubMed PMC

101. Patangia DV, Ryan CA, Dempsey E, Stanton C, Ross RP. Vertical transfer of antibiotics and antibiotic resistant strains across the mother/baby axis. Trends Microbiol 2022;30:47-56. DOI PubMed

102. Nahum GG, Uhl K, Kennedy DL. Antibiotic use in pregnancy and lactation: what is and is not known about teratogenic and toxic risks. Obstet Gynecol 2006;107:1120-38. DOI PubMed

103. Morgan DJ. Drug disposition in mother and foetus. Clin Exp Pharmacol Physiol 1997;24:869-73. DOI PubMed

104. Imoto N, Morita H, Amanuma F, Maruyama H, Watanabe S, Hashiguchi N. Maternal antimicrobial use at delivery has a stronger impact than mode of delivery on bifidobacterial colonization in infants: a pilot study. J Perinatol 2018;38:1174-81. DOI PubMed PMC

105. Stearns JC, Simioni J, Gunn E, et al. Intrapartum antibiotics for GBS prophylaxis alter colonization patterns in the early infant gut microbiome of low risk infants. Sci Rep 2017;7:16527. DOI PubMed PMC

106. Mazzola G, Murphy K, Ross RP, et al. Early gut microbiota perturbations following intrapartum antibiotic prophylaxis to prevent group B streptococcal disease. PLoS One 2016;11:e0157527. DOI PubMed PMC

107. Li X, Stokholm J, Brejnrod A, et al. The infant gut resistome associates with E. coli, environmental exposures, gut microbiome 
maturity, and asthma-associated bacterial composition. Cell Host Microbe 2021;29:975-987.e4. DOI PubMed

108. Pannaraj PS, Li F, Cerini C, et al. Association Between breast milk bacterial communities and establishment and development of the infant gut microbiome. JAMA Pediatr 2017;171:647-54. DOI PubMed PMC

109. Doare K, Holder B, Bassett A, Pannaraj PS. Mother's milk: a purposeful contribution to the development of the infant microbiota and immunity. Front Immunol 2018;9:361. DOI

110. Sakanaka M, Gotoh A, Yoshida K, et al. Varied pathways of infant gut-associated Bifidobacterium to assimilate human milk oligosaccharides: prevalence of the gene set and its correlation with Bifidobacteria-rich microbiota formation. Nutrients 2019;12:71. DOI PubMed PMC

111. Matsuki T, Yahagi K, Mori H, et al. A key genetic factor for fucosyllactose utilization affects infant gut microbiota development. Nat Commun 2016;7:11939. DOI PubMed PMC

112. Murphy K, Curley D, O'Callaghan TF, et al. The composition of human milk and infant faecal microbiota over the first three months of life: a pilot study. Sci Rep 2017;7:40597. DOI PubMed PMC

113. Bäckhed F, Roswall J, Peng Y, et al. Dynamics and stabilization of the human gut microbiome during the first year of life. Cell Host Microbe 2015;17:690-703. DOI PubMed

114. Ahern GJ, Hennessy AA, Ryan CA, Ross RP, Stanton C. Advances in infant formula science. Annu Rev Food Sci Technol 2019;10:75-102. DOI PubMed

115. Gibson GR, Hutkins R, Sanders ME, et al. Expert consensus document: the international scientific association for probiotics and prebiotics (ISAPP) consensus statement on the definition and scope of prebiotics. Nat Rev Gastroenterol Hepatol 2017;14:491-502. DOI PubMed

116. Murphy K, Ross RP, Ryan CA, Dempsey EM, Stanton C. Probiotics, prebiotics, and synbiotics for the prevention of necrotizing enterocolitis. Front Nutr 2021;8:667188. DOI PubMed PMC

117. Beghetti I, Panizza D, Lenzi J, et al. Probiotics for preventing necrotizing enterocolitis in preterm infants: a network meta-analysis. Nutrients 2021;13:192. DOI PubMed PMC

118. Swanson KS, Gibson GR, Hutkins R, et al. The international scientific association for probiotics and prebiotics (ISAPP) consensus statement on the definition and scope of synbiotics. Nat Rev Gastroenterol Hepatol 2020;17:687-701. DOI PubMed PMC

119. Phavichitr N, Wang S, Chomto S, et al; COLOR Study Group. Impact of synbiotics on gut microbiota during early life: a randomized, double-blind study. Sci Rep 2021;11:3534. DOI PubMed PMC

120. Ley RE, Hamady M, Lozupone C, et al. Evolution of mammals and their gut microbes. Science 2008;320:1647-51. DOI PubMed $\mathrm{PMC}$

121. Xie H, Guo R, Zhong H, et al. Shotgun metagenomics of 250 adult twins reveals genetic and environmental impacts on the gut microbiome. Cell Syst 2016;3:572-84.e3. DOI PubMed PMC

122. Kurilshikov A, Medina-Gomez C, Bacigalupe R, et al. Large-scale association analyses identify host factors influencing human gut microbiome composition. Nat Genet 2021;53:156-65. DOI PubMed PMC

123. Bonder MJ, Kurilshikov A, Tigchelaar EF, et al. The effect of host genetics on the gut microbiome. Nat Genet 2016;48:1407-12. DOI PubMed

124. Turpin PL, Ahrens AP, Russell JT, et al. Adolescent autism and autoimmune diagnoses linked to infant gut bacteria whose prevalence is associated with at-risk genetics and/or diet. medRxiv 2021. DOI

125. Murphy K, O'Shea CA, Ryan CA, et al. The gut microbiota composition in dichorionic triplet sets suggests a role for host genetic factors. PLoS One 2015;10:e0122561. DOI PubMed PMC

126. Combellick JL, Shin H, Shin D, et al. Differences in the fecal microbiota of neonates born at home or in the hospital. Sci Rep 2018;8:15660. DOI PubMed PMC

127. Selma-Royo M, Calatayud Arroyo M, García-Mantrana I, et al. Perinatal environment shapes microbiota colonization and infant growth: impact on host response and intestinal function. Microbiome 2020;8:167. DOI PubMed PMC

128. Rodríguez JM, Murphy K, Stanton C, et al. The composition of the gut microbiota throughout life, with an emphasis on early life. Microb Ecol Health Dis 2015;26:26050. DOI PubMed PMC

129. De Filippo C, Cavalieri D, Di Paola M, et al. Impact of diet in shaping gut microbiota revealed by a comparative study in children from Europe and rural Africa. Proc Natl Acad Sci U S A 2010;107:14691-6. DOI PubMed PMC

130. Stearns JC, Zulyniak MA, de Souza RJ, et al; NutriGen Alliance. Ethnic and diet-related differences in the healthy infant microbiome. Genome Med 2017;9:32. DOI PubMed PMC

131. Makino H, Kushiro A, Ishikawa E, et al. Transmission of intestinal Bifidobacterium longum subsp. longum strains from mother to infant, determined by multilocus sequencing typing and amplified fragment length polymorphism. Appl Environ Microbiol 2011;77:6788-93. DOI PubMed PMC

132. Claesson MJ, Wang Q, O'Sullivan O, et al. Comparison of two next-generation sequencing technologies for resolving highly complex microbiota composition using tandem variable 16S rRNA gene regions. Nucleic Acids Res 2010;38:e200. DOI PubMed $\mathrm{PMC}$

133. Hill CJ, Brown JR, Lynch DB, et al. Effect of room temperature transport vials on DNA quality and phylogenetic composition of faecal microbiota of elderly adults and infants. Microbiome 2016;4:19. DOI PubMed PMC

134. Bokulich NA, Chung J, Battaglia T, et al. Antibiotics, birth mode, and diet shape microbiome maturation during early life. Sci Transl Med 2016;8:343ra82. DOI PubMed PMC

135. Vos WM. Microbe Profile: Akkermansia muciniphila: a conserved intestinal symbiont that acts as the gatekeeper of our mucosa. Microbiology (Reading) 2017;163:646-8. DOI PubMed 
136. Coker MO, Laue HE, Hoen AG, et al. Infant feeding alters the longitudinal impact of birth mode on the development of the gut microbiota in the first year of life. Front Microbiol 2021;12:642197. DOI PubMed PMC 